\title{
ARBORIZAÇÃO DO CAMPUS DA UNIVERSIDADE FEDERAL DE SANTA MARIA E CONSCIENTIZAÇÃO DA COMUNIDADE ACADÊMICA
}

\author{
Cibele Rosa Gracioli ${ }^{1}$, Tanny Oliveira Lima Bohner ${ }^{2}$, \\ Cristina Gouvêa Redin ${ }^{3}$, Daniela Thomas da Silva \\ ${ }^{1}$ Eng. Florestal, Dr. Professora Adjunta da Unipampa e do Curso de especialização em Educação Ambiental, \\ Universidade Federal de Santa Maria, RS, Brasil. E-mail: cibelegracioli@gmail.com. \\ ${ }^{2}$ Engenheira Florestal, aluna do programa de especialização em Educação Ambiental pela Universidade Federal de \\ Santa Maria - UFSM. E-mail: tanny.bohner@hotmail.com \\ ${ }^{3}$ Engenheira florestal, Aluna de pós-graduação em Engenharia Florestal, CCR,Universidade Federal de Santa Maria, RS, \\ Brasil. E-mail: crys luanova@hotmail \\ ${ }^{3}$ Engenheira florestal, Aluna de pós-graduação em Engenharia Florestal, CCR,Universidade Federal de Santa Maria, RS, \\ Brasil. E-mail: danyzinha th@hotmail.com
}

\section{RESUMO}

Este trabalho teve como objetivo a conscientização da comunidade acadêmica da Universidade Federal de Santa Maria, RS, no que se refere à importância da utilização de espécies arbóreas adequadas em espaços públicos. A densidade de árvores e a riqueza de espécies foram avaliadas. Foram identificados conflitos na arborização e propostas medidas para solucioná-los. Os seguintes dados foram levantados: espécie arbórea; altura $(\mathrm{m}), \operatorname{CAP}(\mathrm{cm})$; existência ou não de fiação aérea; relação dos exemplares com recuo de edificações $(\mathrm{m})$; existência de elementos arquitetônicos; avaliação do efeito do sistema radicular; e; presença ou ausência de área livre adequada $\left(1 \mathrm{~m}^{2}\right)$. Verificou-se a presença de 1270 exemplares, distribuídos em 75 espécies, 60 gêneros e 32 famílias botânicas. As espécies mais abundantes foram Handroanthus chrysotrichus (Mart. ex DC) Mattos, Peltophorum dubium (Spreng.) Taub., Tipuana tipu (Benth.) Kuntze, Handroanthus heptaphyllus (Vell.) Mattos e Ligustrum japonicum Thunb. A partir deste trabalho, observaram-se conflitos em relação ao porte inadequado de árvores aliados à pequena dimensão de área livre, e a utilização de espécies exóticas em restrição de nativas, que quando utilizadas agregam valor de diversidade a paisagem. A educação ambiental técnica é fundamental na resolução destes problemas, pois promove a conscientização da comunidade a respeito das espécies arbóreas adequadas e da maneira correta de se realizar o plantio em cada situação.

Palavras-chave: paisagismo, conflitos, porte arbóreo, espécies nativas.

\section{ABSTRACT}

This work aimed to raise the academic community, at the Federal University of Santa Maria, regarding the importance of using appropriate tree species in public places. The tree density and species richness were evaluated. Conflicts were identified in afforestation and measures were proposed in order to solving it. The following data were collected: tree species, height $(\mathrm{m})$, CAP $(\mathrm{cm})$, presence or absence of overhead wires; list of examples of buildings with setback $(\mathrm{m})$; 


\section{Monografias Ambientais}

REMOA (Revista Eletrônica do Curso de Especialização em Educação Ambiental da UFSM)

existence of architectural elements, evaluation of the effect of the root system, and, presence or absence of adequate free area $(1 \mathrm{~m} 2)$. There was the presence of 1270 copies, distributed in 75 species, 60 genera and 32 botanical families. The most abundant species were Handroanthus chrysotrichus (Mart. ex DC) Mattos, Peltophorum dubium (Spreng.) Taub., Tipuana tipu (Benth.) Kuntze, Handroanthus heptaphyllus (Vell.) Mattos e Ligustrum japonicum Thunb. Through this work, conflicts related to the size of trees coupled with inadequate small free area, and the use of exotic species on native restriction, when used to add value to the landscape diversity, were observed. Environmental education is a fundamental technique of solving these problems, because it promotes the community awareness concerning the appropriate tree species and the correct way to do the planting in every situation.

Keyword: landscaping, conflicts, arboreal size, native species.

\section{INTRODUÇÃO}

Hoje, um dos principais desafios é interromper o processo de degradação ambiental nas áreas urbanas. Neste sentido, a criação, a recuperação, a qualificação (ou requalificação) dos espaços públicos e de convivência, são fundamentais a sustentabilidade, à valorização da paisagem, à parceria entre instituições públicas e privadas, assim como à melhoria das condições de conforto ambiental.

O plantio de árvores na zona urbana requer cuidados especiais, pois a escolha de uma espécie inadequada pode interferir nas calçadas, no meio-fio, na rede elétrica, nos encanamentos de água e esgoto, e no entupimento de calhas e bocas-de-lobo. Para Soares (1998), uma arborização correta e harmoniosa, ao mesmo tempo em que espelha a cultura e o grau de civilização de uma cidade, constitui-se num dos mais sólidos elementos de sua valorização.

Uma forma de conscientização da população consiste em aproximar do cidadão, os problemas ambientais, sociais, políticos e econômicos do local (ALMEIDA, 2004). Neste contexto, iniciativas em educação ambiental podem trazer soluções para alguns problemas relativos ao meio ambiente.

É por meio da Educação Ambiental que a consciência crítica é despertada e tem como desafio promover a mudança de valores, posturas e atitudes, sendo necessário integrar suas ações aos aspectos ecológicos, políticos, culturais e éticos. A formação do cidadão e a conscientização da sociedade são os elementos essenciais para a execução e êxito dos programas de educação ambiental (KRASILCHIK, 1986).

Deste modo, a pesquisa foi realizada com o objetivo de conhecer, identificar, localizar, catalogar, e divulgar o valor da preservação das espécies arbóreas do Campus da Universidade Federal de Santa Maria (UFSM), em Santa Maria, bem como proporcionar à comunidade a oportunidade de nova postura perante a vegetação, sensibilizando-a quanto à importância da flora, ao meio ambiente e à melhoria da qualidade de vida, aliada à responsabilidade social da Universidade. 


\section{Monografias Ambientais}

REMOA

(Revista Eletrônica do Curso de Especialização em Educação Ambiental da UFSM)

\section{DESENVOLVIMENTO DO TRABALHO}

\section{Caracterização da área de estudo}

O estudo foi desenvolvido no Campus da Universidade Federal de Santa Maria - RS, localizado no Bairro Camobi, a 29 44' 16" de latitude Sul e 533'32" de longitude Oeste. O clima da região, pela classificação de Köppen (MORENO, 1961), enquadra-se como temperado chuvoso e quente, do tipo $\mathrm{Cfa}$. Apresenta uma temperatura média anual de $18,8^{\circ} \mathrm{C}$, e chuvas normalmente bem distribuídas durante todos os meses do ano, contabilizando uma média anual de $1700 \mathrm{~mm}$, sendo os meses de inverno os de maior precipitação (MALUF, 2000). A paisagem natural é caracterizada por uma vegetação campestre e com elementos de floresta estacional semi-decidual (RAMBO, 1956).

\section{Obtenção dos dados}

Os parâmetros levantados foram: a espécie arbórea; com suas variáveis dendrométricas, classe de altura $(\mathrm{m})$ e Circunferência a altura do peito - CAP $(\mathrm{cm})$; existência ou não de fiação aérea; relação dos exemplares com recuo de edificações $(\mathrm{m})$; existência de elementos arquitetônicos em um raio de $2 \mathrm{~m}$; avaliação do efeito do sistema radicular; e; presença ou ausência de área livre adequada $(1 \mathrm{~m} 2)$. Os dados foram coletados em um formulário específico, e após transferidos para o software Microsoft Office Excel ${ }^{\circledR} 2007$ e posteriormente processados no pacote estatístico SAS versão 8.2.

Foram avaliadas todas as árvores pertencentes às principais ruas (Ruas G, H e W) e avenida Roraima, bem como do Parque das Nações, e adjacências de áreas livres para recreação e ajardinamento pertencentes aos prédios dos Centros de Ensino, Reitoria, Casa do Estudante, Restaurante Universitário e Planetário (Figura 1). 


\section{Monografias Ambientais}

REMOA

(Revista Eletrônica do Curso de Especialização em Educação Ambiental da UFSM)

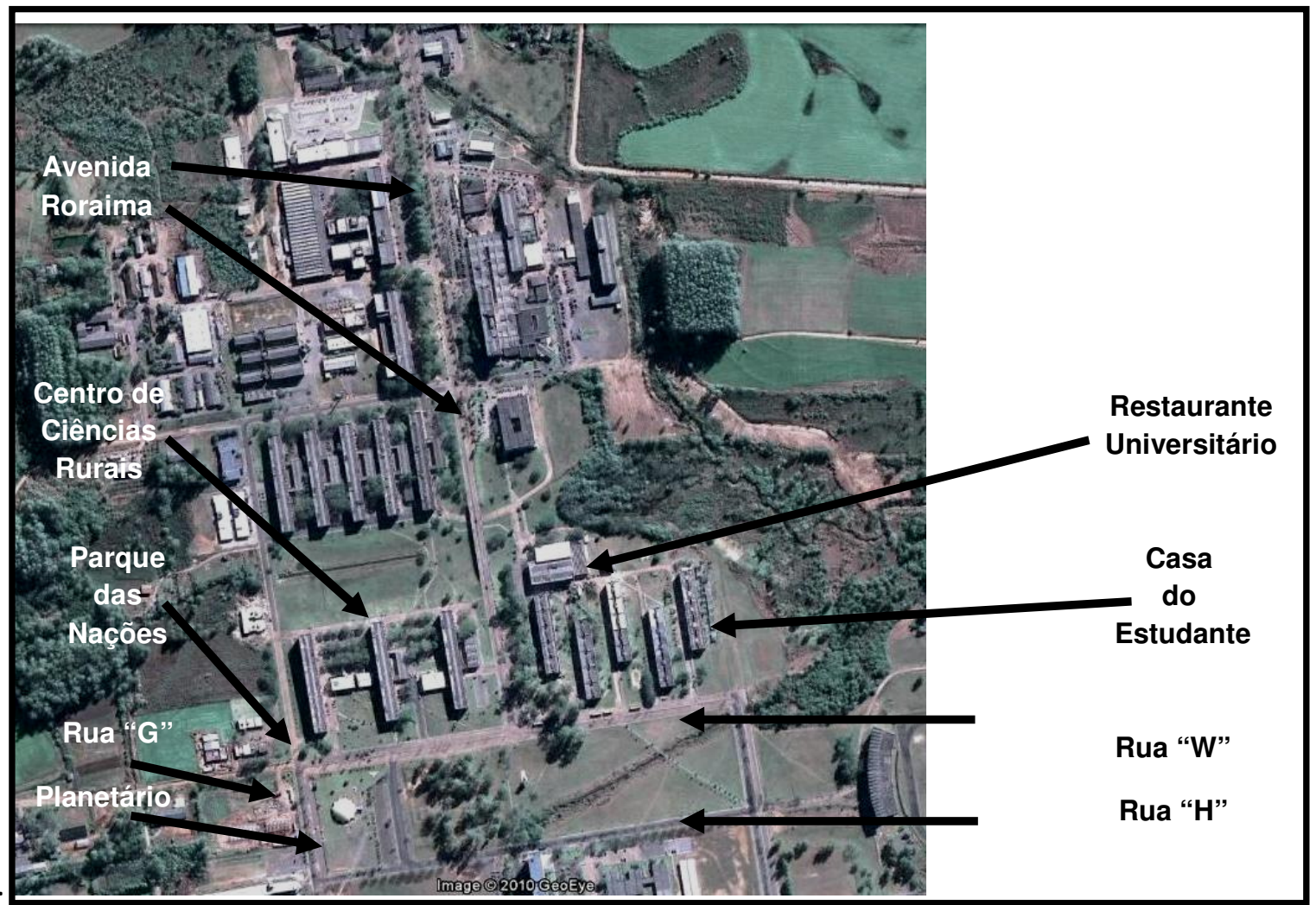

FIGURA 1 - Localização dos pontos levantados, Campus da Universidade Federal de Santa Maria, RS, 2010. Fonte: Google Earth, 2010.

\section{RESULTADOS E DISCUSSÃO}

O presente levantamento indicou a existência de 1270 exemplares, distribuídos em 75 espécies, 60 gêneros e 32 famílias botânicas (Tabela 1). As famílias de maior riqueza foram Fabaceae com 19 espécies, Bignoniaceae com cinco (5) espécies, seguidas de Myrtaceae com quatro (4) espécies, Oleaceae com uma (1) espécie e Arecaceae com cinco (5) espécies.

As espécies mais abundantes foram Handroanthus chrysotrichus (Mart. ex DC) Mattos, Peltophorum dubium (Spreng.) Taub., Tipuana tipu (Benth.) Kuntze, Handroanthus heptaphyllus (Vell.) Mattos e Ligustrum japonicum Thunb., que reuniram $48,81 \%$ de todos os indivíduos. Santos e Teixeira (1990) constataram esse fato ao fazerem o levantamento no Bairro Centro de Santa Maria onde apenas cinco espécies abrangem mais de $70 \%$ de sua arborização. 


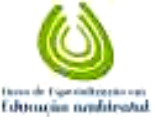

REMOA

\section{Monografias Ambientais}

TABELA 1 - Espécies de árvores encontradas no campus da UFSM, com respectivas famílias botânicas, nome comum, porte, sistema radicular, abundâncias (N) e freqüências (Freq), Santa Maria, 2010.

\begin{tabular}{|c|c|c|c|c|}
\hline Familia & Nome científico & Nome comum & $\mathbf{N}$ & $\%$ \\
\hline Adoxaceae & Viburnum tinus $\mathrm{L}$. & Viburno & 2 & 0,16 \\
\hline \multirow[t]{2}{*}{ Anacardiaceae } & Schinus molle L. & Aroeira-periquita & 10 & 0,79 \\
\hline & Schinus terebinthifolius Raddi & & 3 & 0,24 \\
\hline Araucariaceae & Araucaria angustifólia (Bertol.) Kuntze & Pinheiro-do-paraná & 3 & 0,24 \\
\hline \multirow[t]{5}{*}{ Arecaceae } & Archontophoenix cunninghamii H. Wendl. \& Drude & Falsa -Palmeira-real & 10 & 0,79 \\
\hline & Butia capitata (Mart.) Becc. & Butiá-azedo & 5 & 0,39 \\
\hline & Phoenix canariensis Chabaud & Tamareira & 1 & 0,08 \\
\hline & Phoenix roebelenii O'Brien & Palmeira-fenix & 3 & 0,24 \\
\hline & Syagrus romanzoffiana (Cham.) Glassman & Jerivá & 34 & 2,68 \\
\hline \multirow[t]{5}{*}{ Bignoniaceae } & Jacaranda micrantha Cham. & Caroba & 3 & 0,24 \\
\hline & Jacaranda mimosifolia D. Don & Jacarandá & 9 & 0,71 \\
\hline & Handroanthus albus (Cham.) Mattos & Ipê-branco & 5 & 0,39 \\
\hline & Handroanthus chrysotrichus (Mart. ex A. DC.) Mattos & Ipê-amarelo & 204 & 16,09 \\
\hline & Handroanthus heptaphyllus (Vell.) Mattos & Ipê-roxo & 85 & 6,70 \\
\hline Boraginaceae & Cordia americana (L.) Gottschling \& J.S. Mill. & Guajuvira & 1 & 0,08 \\
\hline Caricaceae & Carica papaya $\mathrm{L}$. & Mamoeiro & 2 & 0,16 \\
\hline Cecropiaceae & Cecropia glaziovi Snethl. & Embaúva & 4 & 0,32 \\
\hline \multirow[t]{3}{*}{ Cupressaceae } & Cupressus sempervirens L. & Cipreste-do-mediterrâneo & 5 & 0,39 \\
\hline & Juniperus chinensis L. & Junipero & 5 & 0,39 \\
\hline & Thuja orientalis L. & Árvore-da-vida-chinesa & 1 & 0,08 \\
\hline Euphorbiaceae & Aleurites fordii Hemsl. & Tungue & 1 & 0,08 \\
\hline \multirow[t]{19}{*}{ Fabaceae } & Acacia podalyriifolia A. Cunn. ex G. Don & Acácia-mimosa & 3 & 0,24 \\
\hline & Anadenanthera colubrina (Vell.) Brenan & Angico-branco & 3 & 0,24 \\
\hline & Ateleia glazioveana Baill. & Timbó & 19 & 1,5 \\
\hline & Bauhinia forficata Link & Pata-de-vaca & 5 & 0,39 \\
\hline & Caesalpinia ferrea Mart. & Pau ferro & 3 & 0,24 \\
\hline & Caesalpinia peltophoroides Benth. & Sibipiruna & 27 & 2,13 \\
\hline & Calliandra bravipes Benth. & Caliandra & 15 & 1,18 \\
\hline & Calliandra tweediei Benth. & Caliandra & 1 & 0,08 \\
\hline & Cassia leptophylla Vogel & Falso-barbatimão & 1 & 0,08 \\
\hline & Enterolobium contortisiliquum (Vell.) Morong & Timbaúva & 18 & 1,42 \\
\hline & Erythrina crista-galli L. & Cortiçeira-do-banhado & 4 & 0,32 \\
\hline & Inga marginata Willd. & Ingá-feijão & 17 & 1,34 \\
\hline & Leucaena leucocephala (Lam.) de Wit & Leucena & 1 & 0,08 \\
\hline & Parapiptadenia rigida (Benth.) Brenan & Angico-vermelho & 3 & 0,24 \\
\hline & Peltophorum dubium (Spreng.) Taub. & Canafístula & 161 & 12,70 \\
\hline & Schizolobium parahyba (Vell.) S.F. Blake & Guapuruvú & 62 & 4,89 \\
\hline & Senna macranthera (DC. ex Collad.) H.S. Irwin \& Barneby & Manduirana & 1 & 0,08 \\
\hline & Senna multijuga (Rich.) H.S. Irwin \& Barneby & Chuva-de-ouro & 2 & 0,16 \\
\hline & Tipuana tipu (Benth.) Kuntze & Tipuana & 85 & 6,70 \\
\hline Fagaceae & Quercus robur (Ten.) A. DC. & Carvalho-europeu & 3 & 0,24 \\
\hline Lauraceae & Persea americana Mill. & Abacateiro & 9 & 0,71 \\
\hline Lythraceae & Lagerstroemia indica $\mathrm{L}$. & Estremosa & 17 & 1,34 \\
\hline \multirow[t]{2}{*}{ Malvaceae } & Ceiba speciosa (A. St.-Hil.) Ravenna & Paineira & 2 & 0,16 \\
\hline & Cedrella fissilis Vell. & Cedro & 2 & 0,16 \\
\hline
\end{tabular}




\section{Monografias Ambientais}

REMOA

(Revista Eletrônica do Curso de Especialização em Educação Ambiental da UFSM)

Continuação da Tabela 1.

\begin{tabular}{|c|c|c|c|c|}
\hline Familia & Nome científico & Nome comum & $\mathbf{N}$ & $\%$ \\
\hline & Brachychiton populneus & Perna-de-moça & 2 & 0,16 \\
\hline & Luehea divaricata Mart. & Açoita-cavalo & 16 & 1,26 \\
\hline Meliaceae & Melia azedarach L. & Cinamomo & 13 & 1,03 \\
\hline \multirow[t]{3}{*}{ Moraceae } & Ficus luchnathiana (Miq.) Miq. & Figueira-do-mato & 3 & 0,24 \\
\hline & Ficus cestrifolia Chodat & Figueira-do-litoral & 1 & 0,08 \\
\hline & Morus nigra L. & & & \\
\hline \multirow[t]{4}{*}{ Myrtaceae } & Eucalyptus spp. & Eucalipto & 44 & 3,47 \\
\hline & Eugenia uniflora $\mathrm{L}$. & Pitangueira & 29 & 2,29 \\
\hline & Myrcianthes pungens (O. Berg) D. Legrand & Guabijú & 1 & 0,08 \\
\hline & Psidium guajava $\mathrm{L}$. & Goiabeira & 16 & 1,26 \\
\hline \multirow[t]{3}{*}{ Pinaceae } & Pinus elliottii Engelm. & Pinheiro & 3 & 0,24 \\
\hline & Pinus patula Schltdl. \& Cham. & Pinheiro-chorão & 3 & 0,24 \\
\hline & Pinus taeda $\mathrm{L}$. & Pinheiro & 16 & 1,26 \\
\hline Platanaceae & Platanus acerifolia (Aiton) Willd. & Platano & 49 & 3,86 \\
\hline \multirow[t]{2}{*}{ Proteaceae } & Grevillea banksii R. Br. & Gravilha-anã & 14 & 1,18 \\
\hline & Grevillea robusta A. Cunn. ex R. Br. & Grevilha & 11 & 0,87 \\
\hline Oleaceae & Ligustrum lucidum W.T. Aiton & Ligustro & 84 & 6,62 \\
\hline Rosaceae & Eriobotrya japonica (Thunb.) Lindl. & Nespeneira & 3 & 0,24 \\
\hline \multirow[t]{3}{*}{ Rutaceae } & Citrus $\times$ limon (L.) Osbeck & Limoeiro & 16 & 1,26 \\
\hline & Citrus reticulata Blanco & Tangerina & 2 & 0,16 \\
\hline & Citrus sinensis (L.) Osbeck & Laranjeira & 8 & 0,63 \\
\hline \multirow[t]{2}{*}{ Salicaceae } & Populus nigra $\mathrm{L}$. & Álamo & 34 & 2,68 \\
\hline & Salix humboldtiana Willd. & Salgueiro & 1 & 0,08 \\
\hline Solanaceae & Solanum mauritianum Scop. & Fumo-bravo & 1 & 0,08 \\
\hline Verbenaceae & Citharexylum montevidense (Spreng.) Moldenke & Tarumã-de-espinho & 1 & 0,08 \\
\hline \multirow[t]{4}{*}{ Taxodiaceae } & Cryptomeria japonica (Thunb. ex L. f.) D. Don & Pinnheiro-do-japão & 3 & 0,24 \\
\hline & Cryptomeria japonica var. elegans-viridis & Pinnheiro-do-japão & 1 & 0,08 \\
\hline & Cunninghamia lanceolata (Lamb.) Hook. & Pinheiro-alemão & 4 & 0,32 \\
\hline & Taxodium distichum (L.) Rich. & Cipreste-do-pantano & 2 & 0,16 \\
\hline \multirow[t]{4}{*}{ Theaceae } & Camelia japonica L. & Camélia & 7 & 0,55 \\
\hline & NI (não identifcada) & & 8 & 0,63 \\
\hline & Mortas & & 10 & 0,79 \\
\hline & TOTAL & & 1270 & 100 \\
\hline
\end{tabular}

Fonte: Lorenzi (2002) e Trópicos (2010).

A distribuição das espécies mais abundantes não foi regular, como se observa na Tabela 1. A grande concentração de Ipê-amarelo (Handroanthus chrysotrichus (Mart. ex DC) Mattos) e Canafístula (Peltophorum dubium (Spreng.) Taub. ) que foi encontrada no campus deve sua elevada abundância aos plantios realizados no início da urbanização do Campus, na década de 70.

A alta densidade da espécie Ipê-amarelo no campus é desaconselhável, dada à susceptibilidade de grandes populações homogêneas à ocorrência de pragas e doenças (SILVA, 2005). Santamour Júnior (2002) afirma que a diversidade de espécies de árvores na paisagem urbana se faz necessário para garantir a máxima proteção contra pragas e doenças. Desta forma, recomenda não exceder mais que $10 \%$ da mesma espécie, $20 \%$ de um gêrnero e $30 \%$ de uma família botânica. Moll (1987) também considera que a freqüência não deverá ultrapassar os $10 \%$ da mesma espécie. 


\section{Monografias Ambientais}

REMOA

(Revista Eletrônica do Curso de Especialização em Educação Ambiental da UFSM)

O predomínio de essências exóticas na arborização de ruas é amplamente observado em vários trabalhos como o desenvolvido na cidade de Pato Branco (PR), onde a arborização da Avenida Tupi foi inventariada tendo como resultado 98 árvores sendo apenas uma nativa (SILVA et al., 2007). Além do Ligustrum japonicum, destacam na tabela 1 espécies que já se encontram na lista de espécies invasoras no Brasil, sendo elas: Grevillea robusta e Eriobotrya japonica (INSTITUTO HÓRUS, 2010).

Outro aspecto polêmico é o uso de espécies frutíferas comestíveis para os humanos. Conforme BIONDI e ALTHAUS (2005) deve-se optar pelo uso de espécies que apresentem frutos pequenos, leves e não carnosos. No entanto, a espécie frutífera nativa Eugenia uniflora, mostra-se promissora no sentido de fornecer alimento a flora e acrescentar valor a biodiversidade.

Os principais problemas encontrados na arborização urbana do Campus da UFSM são apresentados na tabela 2.

Tabela 2 - Influencia do sistema radicular e ocorrência de área livre suficiente das árvores encontradas, com respectivas abundâncias (N)e freqüências (Freq), Santa Maria, 2010.

\begin{tabular}{|c|c|c|c|c|c|c|c|}
\hline \multicolumn{4}{|c|}{ Influência do sistema radicular } & \multicolumn{4}{|c|}{ Ocorrência de área livre suficiente $\left(1 \mathrm{~m}^{2}\right)$} \\
\hline Classe & Infl. Sist Rad. & $\mathbf{N}$ & Freq \% & Classe & Área Livre & $\mathbf{N}$ & Freq \% \\
\hline 1 & NA & 1017 & 80,21 & 1 & AA & 17 & 1,34 \\
\hline 2 & $A C$ & 138 & 10,88 & 2 & $\mathrm{El}$ & 363 & 28,65 \\
\hline 3 & $\mathrm{AC}+\mathrm{MF}$ & 20 & 1,58 & 3 & ES & 887 & 70,01 \\
\hline 4 & AMF & 92 & 7,26 & & & & \\
\hline 5 & APM & 1 & 0,08 & & & & \\
\hline Total & & 1270 & 100 & & & 1270 & 100 \\
\hline
\end{tabular}

$\mathrm{NA}=$ não afeta; $\mathrm{AC}$ = afeta calçada; $\mathrm{AC}+\mathrm{MF}$ = afeta calçada + meio-fio; $\mathrm{APM}$ = afeta prédio ou muro; $\mathrm{AA}=$ área aberta; $\mathrm{EI}=$ Existente $<1 \mathrm{~m}^{2}$ (insuficiente); $\mathrm{ES}=$ Existente $>1 \mathrm{~m}^{2}$ (suficiente)

Sobre a influencia do sistema radicular pode-se dizer que 80,21 \% não afetam por estarem localizadas em áreas de parques ou abertas e, em alguns casos, o porte da árvore pode vir ser proporcional à dimensão da área livre em que ela se encontra. Os casos de "Afetar calçada" $(10,88$ \%) ou "afetar calçada + meio fio" (1,58 \%), são provocados freqüentemente por área livre reduzida para o crescimento dos indivíduos. Em arborização de ruas é aconselhado o uso apenas de espécies cujas raízes cresçam em profundidade (SOUZA, 1994). Ruschel \& Leite (2002) em Lajeado (RS), para um total de 912 indivíduos, não constaram afloramento de raízes em 644 indivíduos, e apenas 268 ocorrências para problemas.

Em relação à área livre, 70,01 \% apresenta "Área livre existente $>1 \mathrm{~m} 2$ " denotando que o problema de conflitos é ocasionado pelo uso indevido de espécies que possuam sistema radicular superficial, não se adaptando á áreas com pavimentação, portanto indicadas para áreas abertas ou de parques. Em Viçosa, Martins et al. (1992), encontraram uma situação diversa, em que predominou dentre quatro condições, a restrita com $21,2 \%$ e com quantidade maior de inexistente $(9,8 \%)$ do que ampla (5,9\%). A área adequada para um bom desenvolvimento das plantas não deve ser inferior a $1,0 \mathrm{~m}^{2}$, em nível de Brasil, pois nos Estados Unidos da América é indicado $6,0 \mathrm{~m}^{2}$, o ideal para uma suficiente aeração e irrigação natural das raízes do vegetal (WYMAN, 1972). 


\section{Monografias Ambientais}

REMOA (Revista Eletrônica do Curso de Especialização em Educação Ambiental da UFSM)

\section{CONCLUSÃO}

As espécies mais abundantes foram Handroanthus chrysotrichus (Mart. ex A. DC.) Mattos, Peltophorum dubium (Spreng.) Taub., Tipuana tipu (Benth.) Kuntze, Handroanthus heptaphyllus (Vell.) Mattos e Ligustrum japonicum Thunb. Os maiores conflitos observados foram em relação ao porte inadequado ao local de implantação aliado a pequena dimensão de área livre, e, a utilização de espécies exóticas em restrição de nativas, que quando utilizadas agregam valor de diversidade a paisagem. Esses conflitos podem ser resolvidos possivelmente com o conhecimento da população acerca do desenvolvimento das espécies arbóreas nativas e exóticas. Nesse contexto, verifica-se a importância da educação ambiental perante a sociedade, uma vez que a mesma pode ser considerada como uma das responsáveis pela melhoria da qualidade de vida da população.

\section{REFERÊNCIAS BIBLIOGRÁFICAS}

ALMEIDA, L.F.R, et al. Educacão ambiental em praças públicas:professores e alunos descobrindo o ambiente urbano. Revista Ciência em Extensão. v.1, n.1, p.91, 2004.

INSTITUTO HÓRUS DE DESENVOLVIMENTO DA CONSERVAÇÃO AMBIENTAL/THE NATURE CONSERVANCY. EsPécies invasoras exóticas: fichas técnicas. 2008. Disponível em: <http://www.institutohorus.org.br/inf_fichas.htm>. Acesso em 16/09/2010.

LORENZI, H. Árvores Brasileiras. V.1. São Paulo: Nova Odessa, 2002. 368 p.

LORENZI, H. Árvores Brasileiras. V.2. São Paulo: Nova Odessa, 2002. 368 p.

KRASILCHIK, M. Educação ambiental no currículo escolar. In: SIMPÓSIO DE EDUCAÇÃO AMBIENTAL, 2, 1987, Santos. Anais....Santos: Museu de Pesca, 1986. p.39-45a.

MALUF, J. R. T. Nova classificação climática do Estado do Rio Grande do Sul. Revista Brasileira de Agrometeorologia, Santa Maria, v. 8, n. 1, p. 141-150, 2000. MARTINS, C. S. Monitoramento da arborização das rua de Belo Horizonte. In: CONGRESSO BRASILEIRO DE ARBORIZAÇÃO URBANA 2, 1994, São Luís.

Anais... São Luís, 1994. p. 421-430.

MORENO, J. A. Clima do Rio Grande do Sul. Porto Alegre: Secretaria da Agricultura, 1961. 42 p.

MOLL, G. Improving the health of the urban forest. American Forest, v.9 , n. - , p. 6 64, 1987.

RAMBO, B. A Fisionomia do Rio Grande do Sul. Porto Alegre:Selbach 1956,. SANTAMOUR JÚNIOR, F. S. Trees for urban planting: diversity uniformity, and ommon sense. In: METRIA CONFERENCE, 7., 990, Lisle. Proceedings. Lisle, p.57-66, 2000.

RUSCHEL, D. LEITE, S. L. C. ARBORIZAÇÃO URBANA EM UMA ÁREA DA CIDADE DE LAJEADO, RIO GRANDE DO SUL, BRASIL Caderno de Pesquisa Série. Biologia, Santa Cruz do Sul, V. 14, N. 1, Jan./Jun. 2002, pp. 7-24

SILVA, L. et al. A. Inventário e sugestões para arborização em via pública de Pato Branco/PR. Revista da Sociedade Brasileira de Arborização Urbana, Piracicaba, 2007. Disponível em: <http:www.revsbau.esalq.usp.br>.Acesso em: $16 / 09 / 2010$.

SOUZA, H.M. de.Algumas espécies nativas para arborização de vias públicas. In: CONGRESSO BRASILEIRO DE ARBORIZAÇÃo URBANA, 2.,1994, São Luis do Maranhão, Anais...São Luis: SBAU, 1994. 613 p. p. 67-74. 


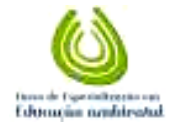

REMOA
GRACIOLI et al., vol.(3), n³, p. 421-429, 2011.

\section{Monografias Ambientais}

(Revista Eletrônica do Curso de Especialização em Educação Ambiental da UFSM)

TROPICOS. Tropicos.org. Missouri Botanical Garden. Disponível em: <http://www.tropicos.org>. Acesso em: 15/09/2010.

WYMAN, D. Parks, malls roadsides: public area plantings. In: Landscape for living -

THE YEARBOOK AGRICULTURE, 1972. Washington: USDA, 1972. p. 77-86. 